ISSN-PRINT 1794-9831 / E-ISSN 2322-7028

Vol. 18 No 3 / sep - dic, 2021 / Cúcuta, Colombia.

do $\underline{\text { https://doi.org/10.22463/17949831.2791 }}$

\title{
Humanización de la atención en salud: análisis del concepto
}

\author{
Humanization of health assistance: concept analysis \\ Humanização da assistência em saúde: análise do conceito
}

\author{
Ángela María Henao-Castaño ${ }^{1^{*}}$ \\ Oscar Javier Vergara-Escobar ${ }^{2}$ \\ Olga Janeth Gómez-Ramírez ${ }^{3}$
}

\section{Resumen}

El análisis del concepto de Humanización de la atención en salud presenta una aproximación teórica y reflexiva, que evidencia los principios y los valores que otorgan un valor agregado a los servicios de salud prestados en un contexto cambiante. Objetivo: Analizar el concepto de Humanización de los servicios de salud. Materiales y métodos: Se aplicó la propuesta por Beth L Rodgers que establece siete pasos para el análisis del concepto. La investigación documental se realizó con bases de datos Springer Plus, Pubmed, EBSCO Host y Biblioteca Virtual en Salud y organismo nacionales; en español, inglés y portugués. Resultados: Con 33 artículos científicos se logra analizar el concepto de humanización de la atención en salud, donde se describen atributos éticos, epistemológicos y metodológicos. De igual forma, se resalta la comunicación asertiva y efectiva en la atención en salud, en el contexto de la salud-enfermedad, aportando a la enfermería concepciones sobre humanización en ese marco. Conclusiones: La humanización de la atención en salud permite desarrollar estrategias en beneficio del bienestar y la buena práctica. Aunque el concepto de humanización de la atención se puede analizar desde diferentes perspectivas, la metodología de análisis de concepto es una perspectiva útil para considerar a la humanización de la atención en salud como un eje orientador de políticas de mejoramiento y calidad de la atención, así como en la promoción de prácticas que faciliten el trabajo colaborativo, la comunicación y el desarrollo humano.

Palabras clave: Humanización de la atención; atención de enfermería; análisis del concepto; enfermería.

\begin{abstract}
Autor de correspondencia*
1* Doctora en enfermería. Profesora Asociada. Facultad de Enfermería Universidad Nacional de Colombia. Correo: angmhenaocas@unal.edu.co (i) 0000-0003-4203-0016

${ }^{2}$ Doctor en enfermería. Profesor Agregado Escuela de Enfermería, Fundación Universitaria Juan N Corpas. Bogotá. Correo: oscar.vergara@juanncorpas. edu.co (D) 0000-0003-3158-9017

${ }^{3}$ Doctora en enfermería de la Universidad Nacional de Colombia, Bogotá - Colombia. Profesora asociada a la Facultad de Enfermería de la Universidad Nacional de Colombia. Correo: ojgomezr@unal.edu.co (i) 0000-00029160-4170
\end{abstract}

Recibido: 28 febrero 2021

Aprobado: 6 julio 2021

\section{Para citar este artículo}

Henao-Castaño AM, Vergara-Escobar OJ, Gómez-Ramires OJ. Humanización de la atención en salud: análisis del concepto. Rev. cienc. cuidad. 2021; 18(3):74-85. https:// doi.org/10.22463/17949831. 2791

(C) Universidad Francisco de Paula Santander. Este es un artículo bajo la licencia CC-BY-NC-ND

\begin{abstract}
The concept analysis of Humanization of health assistance presents a theoretical and reflective approach, evidencing the principles and values that give an aggregated value to the health services provided in a changing context. Objective: Analyze the concept of Humanization of health services. Materials and Methods: The proposal of Beth L. Rodgers was applied, which establishes seven steps for concept analysis. The documentary research was made with the databases Springer Plus, Pubmed, EBSCO Host and Biblioteca Virtual en Salud (Virtual Library of Health) and national organizations; in Spanish, English and Portuguese. Results: It was possible to analyze the concept of humanization of health assistance with 33 scientific articles, where ethical, epistemological and methodological attributes are described. Similarly, the assertive and effective communication is emphasized for health assistance, in the context of health-disease, contributing to nursing conceptions about humanization in that framework. Conclusions: Humanization of health assistance allows the development of strategies for
\end{abstract}




\section{Scientific Journal of Nursing}

the benefit of well-being and the good practice. Although the concept of humanization of assistance can be analyzed from different perspectives, the methodology of concept analysis is a useful perspective to consider the humanization of health assistance as a central concept that orientates policies of improvement and quality of assistance, as well as the promotion of practices that facilitate collaborative work, communication and human development.

Keywords: Humanization of assistance; nursing assistance; concept analysis; nursing.

\section{Resumo}

A análise do conceito de humanização da assistência em saúde apresenta uma contribuição teórica e refletiva, que evidencia os princípios e os valores que somam valor aos serviços de saúde prestados em contextos mutáveis. Objetivo: Analisar o conceito de humanização nos serviços de saúde. Materiais e métodos: Aplicou-se a proposta de Beth L Rodgers que estabelece 7 passos para a análise de conceito. A pesquisa documental realizou-se nas bases de dados: Springer Plus, Pubmed, EBSCO Host, Biblioteca virtual em saúde e organismos nacionais; em espanhol, inglês e português. Resultados: Com 33 artigos científicos logrou-se analisar o conceito de humanização da assistência em saúde, descrevendo atributos éticos, epistêmicos e metodológicos. Igualmente, ressaltou-se a comunicação assertiva e efetiva no atendimento em saúde, no contexto de saúde-doença, contribuindo à enfermagem e conceitos de humanização nesse marco. Conclusões: A humanização da assistência em saúde permite desenvolver estratégias em beneficio do bem estar e boa pratica. Embora o conceito de humanização do atendimento possa se analisar desde diferentes perspectivas, a metodologia de analise do conceito é uma ferramenta útil para considerar à humanização da assistência em saúde como eixo orientador de politicas de melhoramento e qualidade do atendimento, assim como na promoção de práticas que facilitem o trabalho colaborativo, a comunicação e o desenvolvimento humano.

Palavras-chave: Humanização da assistência; cuidados de enfermagem; estudo de prova de conceito; enfermagem.

\section{Introducción}

El Grupo de Cuidado de la Facultad de Enfermería de la Universidad Nacional de Colombia, plantea una metodología de análisis de concepto, basado en los pasos propuestos por Beth Rogers. El análisis del concepto de humanización de la atención en salud, se efectuó a partir de la siguiente pregunta ¿Cuáles son los elementos éticos, epistemológicos y metodológicos de la humanización de los servicios de salud?. En este sentido, se propone un análisis reflexivo, basado en la evidencia disponible sobre el tema de humanización de los servicios de salud alrededor del mundo.

La humanización lleva implícito un sistema complejo de organización y de civilización que respeta la autonomía de los individuos, la diversidad de las ideas, la libertad de expresión y el rescate de la subjetividad. El desafío de los profesionales de la salud y en especial deylos de enfermería es tener claridad de los usos del concepto y su implementación a la práctica del cuidado, frente al progreso técnico - científico; por tanto, este uso del concepto recae directamente en los sujetos de cuidado y la sociedad, en función de la inclusión de todas las personas y pueblos en beneficio de este progreso $(1,2)$.

El Ministerio de Salud define la humanización en un contexto de calidad, como un principio orientador de la atención integral en salud; hace referencia a la ob- 
ligación que tienen los actores que intervienen en dicha atención de garantizar la accesibilidad, la oportunidad, la pertinencia, la seguridad y la continuidad en la atención a gestantes, niñas, niños y adolescentes (GNNA). Hay que mencionar además, que se debe respetar su condición y dignidad humana, reconociendo su contexto socio cultural, la diversidad poblacional y territorial que se expresa en la particularidad de su desarrollo (3).

El análisis del concepto es una vía para el desarrollo de la teoría, proporcionando una oportunidad para explicar y describir fenómenos de interés útiles a la práctica. Es además un ejercicio lingüístico que sirve para determinar sus atributos y aunque es un proceso riguroso y preciso, el producto final siempre será tentativo (4). Los conceptos son palabras que describen: objetos, propiedades, eventos y relaciones entre los llamados términos descriptivos (5). El interés en la definición de conceptos en enfermería tiene por objetivo conseguir la precisión en sus términos, para facilitar y ampliar la comprensión entre sus agentes y la importancia de percibir el sentido de las palabras y el vínculo con su historia u origen; es decir, representarlas a través de símbolos y significados establecidos por el uso, la costumbre o la asociación (6).

Chinn y Kramer definen al concepto como "una formulación mental compleja de experiencia empírica"; por ende, los conceptos son "representaciones" cognitivas abstractas de realidades perceptibles que se forman por medio de experiencias directas e indirectas. Van desde observaciones empíricas observables directamente hasta inferencias mentales visibles indirectamente y relativamente abstractas (7).

El propósito de este artículo es presentar el análisis del concepto de humanización de la atención en salud por medio de la metodología del concepto, siguiendo los pasos propuestos por Rodgers (Ver Tabla 1).

\section{Materiales y Métodos}

El enfoque de investigación al que corresponde este estudio es el análisis de concepto, el mismo se llevó a cabo bajo los siete pasos planteados por Rodgers y Knafl (8), los cuales permitieron: identificar el concep- to de interés, los términos sustitutos, ejemplo y fecha de colección, los atributos del concepto, las referencias, antecedentes y consecuencias del concepto, la relación de los conceptos y el modelo de caso; igualmente, se efectuaron comparaciones interdisciplinarias y temporales como se describe en la Tabla 1. En este análisis de concepto se consultaron artículos que incluyeran las palabras claves indexadas en bases de datos como lo son: Springer Plus, Pubmed, EBSCO Host y Biblioteca Virtual en Salud; así como la página del Ministerio de Salud y Protección Social de Colombia.

Para el concepto de Humanización de los servicios de salud, los términos descriptores en Ciencias de la Salud fueron consultados en tres idiomas (inglés, portugués y español): Humanization of Assistance, Humanização da Assistência, humanización de la atención. En consecuencia el término se lo define como el tipo de asistencia que corresponde a un tratamiento más efectivo, por cuanto el paciente es escuchado y respetado por los profesionales de la salud. Se debe agregar, que es necesario humanizar las condiciones de trabajo de estos profesionales. Biblioteca virtual en Salud, Términos Descriptores en Ciencias de la Salud DeCS).

Al mismo tiempo, se encontraron artículos que presentaron el concepto a partir de su uso y relevancia; de igual forma, se identificaron palabras con sinónimos como: el cuidado humanizado, atención humanizada, salud humanizada, comprensión del ser humano, integralidad del sujeto, dignidad y condición humana. Estos términos permitieron rastrear 20 piezas de investigación que evidencian un panorama amplio, diverso y concreto del uso respecto al concepto sobre humanización en la atención de enfermería.

Dentro de los criterios de inclusión de la búsqueda se seleccionaron artículos publicados en los últimos 20 años, en los idiomas inglés, portugués, español y su disponibilidad en texto completo; en definitiva, se seleccionaron artículos de investigación, revisiones sistemáticas y repositorios documentales. En función de los criterios planteados anteriormente se obtuvo una selección definitiva por su relevancia y contenido de 33 artículos y documentos presentes en páginas de entes gubernamentales como el Ministerio de Salud y Protección Social. 


\section{Scientific Journal of Nursing}

Tabla 1. Pasos modelo de Rogers. Análisis del concepto de Humanización de los servicios de salud

\begin{tabular}{|c|c|}
\hline Pasos & Concepto de Humanización de los servicios de salud \\
\hline 1. Identificar el nombre del concepto de Interés. & Humanización de los servicios de salud \\
\hline $\begin{array}{l}\text { 2. Identificar términos sustitutivos y usos rele- } \\
\text { vantes del concepto }\end{array}$ & $\begin{array}{l}\text { Cuidado Humanizado } \\
\text { Atención Humanizada } \\
\text { Salud Humanizada } \\
\text { Comprensión del ser humano. } \\
\text { Integralidad del sujeto } \\
\text { Dignidad y Humanizar } \\
\text { Condición humana }\end{array}$ \\
\hline $\begin{array}{l}\text { 3. Identificar y seleccionar un ámbito/muestra } \\
\text { apropiada para la colección de los datos }\end{array}$ & $\begin{array}{l}\text { Base de Datos de: Springer Plus, Pubmed, EBSCO Host y } \\
\text { Biblioteca Virtual en Salud } \\
\text { Años } 2000-2020\end{array}$ \\
\hline 4. Identificar atributos del concepto. & $\begin{array}{l}\text { Atributos éticos, autonomía del paciente, solidaridad con la } \\
\text { persona } \\
\text { Principios Éticos } \\
\text { Atributos epistemológicos, Humanismo y Existencialismo } \\
\text { Atributos metodológicos y prácticas que Humanizan la at- } \\
\text { ención en salud } \\
\text { La comunicación asertiva y efectiva en el proceso de cuidar } \\
\text { humanizado } \\
\text { Seguridad del paciente } \\
\text { Políticas y programas de humanización } \\
\text { Satisfacción del usuario y la familia }\end{array}$ \\
\hline $\begin{array}{l}\text { 5. Identificar referencias, antecedentes y conse- } \\
\text { cuencias del concepto }\end{array}$ & El desarrollo de habilidades emocionales \\
\hline $\begin{array}{l}\text { 6. Identificar conceptos relacionados con el } \\
\text { concepto de interés. }\end{array}$ & $\begin{array}{l}\text { Humanización } \\
\text { Dignificación del ser humano }\end{array}$ \\
\hline 7. Identificar un caso modelo del concepto. & Ejemplo aplicado a la práctica, experiencia. \\
\hline
\end{tabular}

Fuente: Elaboración propia sobre análisis del concepto (2021).

\section{Resultados}

A continuación, se describen los siete pasos planteados por Beth Rogers para el análisis del concepto sobre la humanización de la atención en salud.

\section{Paso 1. Identificar el nombre del concepto de Interés.}

El primer paso es un pilar para avanzar en el proceso de análisis del concepto, se parte del concepto de la humanización por el cual se "resalta la acogida (hospitalidad), el diálogo, el apoyo espiritual y emocional, la escucha activa, el consuelo ante la tragedia que suele ser la enfermedad"(9). Coincide con la anterior definición y afirma que todos los ciudadanos deben recibir una atención humanizada y sensible a sus necesidades, incluyendo las exigencias formales para contar con un sistema de información y atención, que respete la dignidad y la autonomía de la persona.
Por otra parte, la humanización puede ser considerada como un eje fundamental dentro de las ciencias de la salud, puesto que incluye directrices y prioridades a todas las personas y procesos, proponiendo transformaciones en las relaciones sociales de los actores participantes y en las interacciones que se generan entre ellos. Del mismo modo, se busca la implementación de instancias científicas, estrategias de intervención y atención, dentro de las que se establece el trabajo en equipo y la atención de las personas desde diferentes disciplinas y campos de acción; igualmente se valoriza la participación de los actores y la corresponsabilidad de gestores, trabajadores y usuarios en el campo de dicha atención. En consecuencia, la "Humanización en salud debería ser una base fundamental en la relación con seres humanos; sin embargo, actualmente se viene observando un fenómeno de distanciamiento terapéutico, asociado en algunos casos a la negligencia 
y despersonalización de las relaciones, lo que permite reconocer que hay procesos de deshumanización que se han ido generalizando de manera sistemática. Para Canizales y cols. los relacionan con los desarrollos tecnológicos, pero también esto podría generarse por los diferentes sistemas de salud, que no cubren las necesidades y expectativas de los enfermos/as" concepto que deja ver una realidad de un sistema de salud, dentro del paradigma biológico, haciendo de este concepto un reto (9).

Al hablar de humanización de la atención en salud es significativo tener en cuenta todo un conjunto de valores implicados en esta; en primer lugar, las instituciones del sector salud, tienen el compromiso no solo de proveer al recurso humano, los mecanismos para efectuar apropiadamente sus competencias, atenuar sus debilidades formativas y de comunicación (10); en segundo lugar, estos factores suponen una intervención adecuada sobre las limitaciones en infraestructura y equipamiento que influyen negativamente en el logro de una atención con calidad (11) y la satisfacción del paciente (12).

Paso 2. Identificar términos sustitutivos y usos relevantes del concepto.

La literatura científica, permitió en este ejercicio identificar términos sustitutos que en el tiempo tuvieron una trayectoria importante desde su origen, desarrollo y evolución en las ciencias de la salud y en el actuar del ser humano en diversas situaciones de la salud, logrando identificar con estos conceptos la significancia, el uso y la aplicación. que en algunos casos es fuerte y en otros depende del contexto en donde se da relevancia a la humanización. En este orden de ideas los términos sustitutivos son los siguientes: Cuidado Humanizado, Atención Humanizada, Salud Humanizada, Comprensión del ser humano, Integralidad del sujeto, Dignidad y Condición humana $(13,14)$.

Al hablar de Atención Humanizada, siempre se evoca a un concepto que se ha utilizado constantemente en el sector de la salud. Hay autores que sitúan la humanización como la búsqueda de atención, además de la técnica y la preocupación por la enfermedad y la consideran como la necesidad de evaluar al ser humano teniendo en cuenta sus características personales. De igual forma, se han observado argumentos que se refieren a la modificación de determinadas prácticas, principalmente en relación con la mejora y cualificación de la atención a través del profesional sanitario, con el objetivo de hacerla humanizada (15).

Así que este análisis permite dimensionar una reflexión sobre lo que es el acto de atención en servicios de salud y por qué en los últimos años se ha convertido en una preocupación no sólo para los profesionales de la salud, sino, también para la sociedad y los sistemas de salud de la región; siendo, un eje norteador en las políticas de salud se atribuye a este término como características esenciales que es mandatorio y ejecutorio brindar atención humanizada en servicios de salud (16).

En cuanto a el Cuidado Humanizado, Souza y Cols. hacen referencia a que las concepciones sobre humanización remiten a las cuestiones sociales, que muestran la relación entre el profesional y el usuario en la atención; así como a las cuestiones gerenciales, que muestran las dificultades y facilidades en el ámbito asistencial para una asistencia humanizada (17). Situación que sitúa al concepto desde un ángulo social, administrativo y de relación de seres humanos, que tiene un patrón de comportamiento y de interrelación donde la comunicación va más allá de un emisor y un receptor ante la prestación de servicios en salud. Además, es importante que los profesionales de enfermería otorguen cuidados centrados en los usuarios y que estos cuidados humanizados se integren a la praxis para mejorar la calidad de la atención en los servicios de salud (18, 19).

Hay que mencionar, además, que la enfermería se ha apoyado en teorías que aportan aspectos significativos a la ciencia del cuidado, entre las que se destaca la teoría del cuidado humanizado de Jean Watson, mundialmente reconocida por el proceso caritas, que vincula los valores propios de la enfermería desde una perspectiva cualitativa como arte y la ciencia del cuidado. Por su parte, Kristen Swanson propone el conseguir un bienestar, que se humanice el cuidado por parte de los profesionales con amplia experiencia, habilidad y capacidad en el conocimiento integral y holístico de la persona en su cotidianidad, ya que todos los aspectos que rodean su vida y su contexto intervienen de forma positiva o negativa en su bienestar (20).

En cuanto a la Salud Humanizada se debe analizar desde la Inter profesionalidad, puesto que los equipos de salud están trabajando en pro del bienestar de las personas, familias y comunidades; de allí la importancia de la concepción de la salud dentro de una sociedad, lo que permitirá establecer una relación que se debe dar entre los profesionales de la salud y las personas que transitan por estados de alteración de su bienestar. Olivera recuerda que la inclusión de otros profesionales de la salud, así como de los gerentes, es importante porque facilita la validación de la humanización; no obstante, es el profesional de enfermería a quien se le considera indispensable para la realización de la atención humanizada (15). 
Desde la Comprensión del ser humano se ha interpretado que los profesionales de la salud deben estar preparados para interpretar, leer y ayudar a otros humanos a partir de la comunicación como eje fundamental en el proceso humano y más cuando se trate del bienestar; al respecto Medina (15) citado por Olivera, afirma que la buena comunicación es un factor importante para el cuidado humanizado, ya que es un gran vehículo por el cual se puede practicar la humanización. Por lo tanto, parece ofrecer apoyo emocional, acceso a la información y hablar con el cliente, animándolo a expresar sus sentimientos, haciendo que el cuidado sea más humanizado. Con este vehículo de habilidades comunicativas el enfermero del futuro puede escalar niveles que sirvan para dimensionar las necesidades del ser humano, basadas en una atención centrada en la persona, el contexto, la familia y sus situaciones de salud; situaciones que conforman un mundo particular único, permitiendo una interacción continua lo que facilitará una atención humanizada en cualquier escenario de la salud humana (21). Como se puede evidenciar en estos escenarios se reclama la humanización de los servicios de salud; de ahí, que el profesional de enfermería quien es el responsable de cuidar con arte y ciencia, debe hacer uso de todos los medios para ayudar a las personas en los procesos de promoción, prevención, recuperación y rehabilitación de la salud (22).

La Integralidad del sujeto, es un concepto sustitutivo, pero a la vez diciente de lo que las organizaciones de salud deben tener en los modelos de atención, puesto que el cuidado holístico es una marcada tendencia en la actualidad. Se debe agregar, que después de mucho tiempo marcado por hegemonías se fracturó la atención de la persona por especialidades (23). En este sentido se recuerda un episodio de distancia que se tomaba en la atención de las personas, distancia que emerge entre el sentido común y el conocimiento científico, especialmente cuando el individuo se inserta en un contexto de cuidado y hospitalario (24). Postura que desencadena en sentimientos como impotencia, ansiedad, estrés y miedo con lo que le pueda suceder, dando origen a varias preocupaciones tanto para el sujeto mismo como para sus familiares, manteniendo así una posición sumisa de cuidado, porque se sienten en condiciones desiguales en comparación con los profesionales (considerados como poseedores del conocimiento científico) (25).
Es aquí donde las oportunidades de encuentros frente a las distancias siempre se deben contemplar en los equipos de salud, en función de la consciencia del ser, estar y ayudar a otro ser humano, basado en la comunicación, la empatía y sobre todo el respeto digno a la persona.

Dignidad y Humanizar, son quizás los conceptos por los cuales se debe preocupar siempre un profesional de la enfermería a fin de mantener una relación eficiente en la atención en salud dentro de los servicios; y es desde allí donde cada actor debe poner de su parte para comprender que más que una transacción, debe existir un vínculo humano que dignifique a la persona que transita por su estado de salud y al profesional que da todo su potencial para actuar; por tanto, el humanizar se definiría como el reconocer a las personas que buscan en los servicios la resolución de sus necesidades sanitarias, como sujetos de derechos, dotados de su individualidad y autonomía; por tanto, se debe siempre haciendo insistir en que algunos principios éticos deben ser considerados por el equipo en la relación con el usuario (15).

En definitiva estos términos sustitutivos, aunque sean diferentes en su concepción tienen un fin común, como es la humanización de la atención en salud, respecto a los marcos políticos, los contextos clínicos o en salud pública, los educativos e investigativos, a fin de establecer una relación reciproca e integral, a partir de un cuerpo de conocimientos epistemológicos

Paso 3. Identificar y seleccionar un ámbito/muestra apropiada para la colección de los datos.

Conviene subrayar que el concepto de humanización de los servicios de salud ha evolucionado a través de diferentes contextos sobre el actuar de la salud humana, partiendo del concepto de humanización en la esfera del trabajo de la enfermería, adaptándola en la actualidad a escenarios específicos de atención en salud como: la salud pública, servicio de urgencias y unidad de cuidado intensivos, atención a pacientes con enfermedades con Virus de Inmunodeficiencia Humana (VIH) y COVID 19 (26) (Ver tabla 2). 


\section{Scientific Journal of Nursing}

Tabla 2. Desarrollo del concepto de Humanización de los servicios de salud

\begin{tabular}{lc}
\hline \multicolumn{1}{c}{ Ámbito desarrollo del concepto } & año \\
Humanización del trabajo de enfermería & 2003 \\
Humanización en salud-Humanización de profesionales y usuarios & 2004 \\
Humanización en cuidado de la salud & $2005-2006$ \\
Humanización en enfermería & 2008 \\
Humanización en salud pública & 2013 \\
Humanización servicios de salud & 2013 \\
Humanización en la Unidad de cuidado Intensivo & 2014 \\
Comunicación como instrumentos de humanización & 2016 \\
Humanización de la atención en salud & 2018 \\
Humanización servicios de urgencias & 2019 \\
Humanización en paciente con enfermedad COVID 19 & 2020 \\
\hline
\end{tabular}

Fuente: Elaboración propia sobre análisis del concepto (2021)

La humanización de los servicios de salud se encuentra circunscrita a los diferentes ambientes de cuidado, al respecto se encontraron estudios en los servicios de urgencias en donde la asistencia humanizadora comprende: ofertas de servicios y tecnologías, recursos humanos y materiales e infraestructura, orientadas a una atención segura con garantía de confort y bienestar a los usuarios de los servicios de salud (17). Se sabe que las tecnologías son dispositivos útiles para modificar radicalmente el proceso de trabajo, especialmente si los profesionales están dispuestos a utilizar su "caja de herramientas", con el objetivo de resolver las necesidades de salud de los usuarios como conductas de humanización (27).

Se debe agregar que en los servicios de terapia intensiva los enfermeros tienen una comprensión aparentemente intuitiva del concepto de humanización, partiendo de una perspectiva de empatía por el usuario. La empatía es una herramienta valiosa en el proceso de humanización, en donde el individuo se coloca en el lugar del otro, actuando según ideales y valores, calificando el cuidado brindado (28).

La asistencia en salud debe resolver de manera eficaz los problemas de salud; por tanto la asistencia hacia el usuario es fundamental para su humanización. La ética del cuidado merece una atención similar y cubre la posibilidad concreta de que los pacientes sigan los tratamientos prescritos (ontología del cuidado). La esfera estética es otra faceta a considerar porque integra la atención que se le da a las condiciones ambientales, la higiene y limpieza, a la identificación nominal de los pacientes y sus acompañantes, entre otros (29).

El propósito u objetivo de humanizar en salud, implica aceptar y reconocer que en este ámbito y en sus prácticas se pueden presentar graves problemas y deficiencias de muchas de las condiciones dadas, por la definición del diseño, la organización y la implementación de la atención de la salud, entendida desde la humanidad, tanto por organismos, prácticas estatales y sociedad civil (21).

Humanizar desde la perspectiva de la enfermería es vista como la humanización del cuidado de enfermería, a la que se le debe sumar la competencia técnica y científica; de igual manera, se la considera como una ética que considera y respeta la singularidad propia sobre las necesidades del usuario y del profesional en los sistemas de salud (30), pero que además acepta los límites de cada participante en la situación que requiere la producción de actos en Salud (31). La humanización de la asistencia de enfermería debe ser vista como la participación del sujeto que, siendo también humano, es capaz de expresar actitudes humanas e "inhumanas" basadas en relaciones entre todos en la vida cotidiana (32).

\section{Paso 4. Identificar atributos del concepto.}

Los atributos del concepto de humanización de la atención en salud, implica la comprensión del ser humano en su proceso de vivir, en sus derechos, en su especificidad y en su integridad. Orientarse por el cuidado es romper con la lógica de formación excesivamente basada en la hegemonía biomédica, en el autoritarismo de las relaciones, en el poder, construido a partir de un saber que silencia a los otros y cosifica a los sujetos (11). Uno de los atributos que presenta el concepto, está relacionado con la comunicación como instrumento básico en el proceso de la atención humanizada con respecto al paciente hospitalizado. La comunicación en enfermería ejerce un papel importante en lo que se refiere a un cuidado competente y humanitario (10); por consiguiente, se debe privilegiar al paciente a través de una relación terapéutica, entendida como un proceso interactivo y personalizado, en donde se privilegie la afinidad y la comprensión (33). En consecuencia la acción comunicativa promueve la conexión y la coordinación de la atención e in- 
cluye todos los tipos de tecnologías, porque la acción comunicativa es la única capaz de articular los diferentes mundos y sus respectivos tipos de acción (34).

A su vez se considera que la humanización en la atención se enfatiza en las instituciones de salud, y la comunicación permite al equipo comprender las necesidades del paciente en su condición de vulnerabilidad por la enfermedad; razón por la cual en el proceso de la hospitalización surge la necesidad de reflexionar respecto a la relevancia de la comunicación en el cuidado humanizado en enfermería. Se debe resaltar que la comunicación, como instrumento para la humanización de la asistencia al paciente, debe ser percibida, tanto a partir del lenguaje verbal como no verbal, ya que el sistema verbal permite al profesional de enfermería en la relación con el paciente, percibir características del no-verbal, reveladas en señales, gestos y movimientos que expresan mensajes y así comprender las necesidades reales del paciente.

Con frecuencia, la individualidad de cada paciente se silencia, no existiendo un espacio para su cuidado que reconozca sus miedos, inseguridades, preocupaciones, necesidades, angustias e incertidumbres o incluso que no se garantice la participación del paciente como un individuo autónomo, que tenga libertad de expresar lo que siente, percibe y piensa sobre su condición de ser enfermo (33). Por tanto, humanizar significa acoger al paciente en su esencia, a partir de una aportación traducida en la solidaridad, en la comprensión del ser enfermo en su singularidad y en la apreciación de la vida.

Es necesario recalcar que la práctica sin la comprensión de los atributos del paciente, sólo lleva a la realización de la atención en una relación vertical-unidireccional entre el profesional y el usuario. Por ese motivo los atributos indispensables en los actos del cuidado humanizado deben estar relacionados con el ser humano, la relación profesional usuario, sujeto del cuidado, comunicación y enfoque holístico (35).

Por otra parte, la percepción de las enfermeras, en cuanto a la ampliación del ámbito competencial, implica cambios que no todos los profesionales están dispuestos a asumir, ya sea por cuestiones legales, conceptuales, deontológicas o de solapamiento de roles con otros profesionales que históricamente han gozado de mayor reconocimiento social (36).

Paso 5. Identificar referencias, antecedentes y consecuencias del concepto.

Para que la humanización en la atención en salud sea efectiva en la práctica es necesario el trabajo en equipo, valorando y realizando la inclusión de todos los sujetos protagonistas de los centros de salud (37).

\section{Antecedentes del concepto}

La situación del paciente, las habilidades comunicativas del personal de salud y la condición de ambos, como seres hu- manos, influyen en las palabras, gestos y actitudes durante la relación enfermera-paciente, en donde la presencia, lo que se hace y la forma de hacerlo dejan una importante huella en los pacientes y sus familiares (38).

\section{Consecuencias}

En efecto, en el medio hospitalario, es necesario que se desarrollen habilidades emocionales y que permitan sensibilizarse con las situaciones vivenciadas cotidianas, evitando el tecnicismo; desde este punto de vista es necesario ofrecer un cuidado humanizado al cliente, evitando siempre la desconfianza. Se deben romper los estereotipos históricos que han padecido las enfermeras y así se dé espacio para que se produzca una mejora de su visión social (39).

Es oportuno destacar que la humanización del cuidado en la vida, respetando la individualidad de la persona, debe suscitar una percepción holística sobre el ser humano, extrapolando la comprensión "biológica de la enfermedad y contemplando los aspectos psicológicos, sociales y espirituales que directa o indirectamente influencian en el proceso de salud" (33).

Paso 6. Identificar conceptos relacionados con el tema de interés.

En este paso es fundamental relacionar las piezas científicas y el concepto principal de estudio, desde la postura de Pallares quien afirma que la humanización está dirigida a la reivindicación de los derechos y de los deberes, a la autonomía y al respeto de la voluntad, propiciando cada vez más el bienestar físico y mental de los pacientes, atendiéndolos de manera integral, teniendo en cuenta que son seres completos, totalitarios, únicos e irrepetibles que se encuentran involucrados en un contexto particular. Además, se debe tener presente su sentir, pensamientos e ideas, ya que actúan de acuerdo con un conjunto propio de valores, creencias, prácticas, características y experiencias vividas a lo largo de la vida, lo que los hace mucho más sensibles de lo que a simple vista es observable; es decir, se requiere una visión y un cuidado holístico ayudando a la prolongación de su vida (40).

Este análisis de concepto de la humanización de la atención en salud tiene una íntima relación con habilidades blandas como la empatía, la compasión, el trato digno (21), la solidaridad, la fraternidad, el afecto, el amor y el respeto por el otro. (23). Se debe agregar, que todo esto está centrado en el vehículo de la comunicación (15) que es quizás la herramienta humana más vigente para reconocer las necesidades en salud. De ahí, que se deba promover la formación continua en las universidades, concentrando la atención en este proceso fundamental que tienen los humanos, que les sirve para dar a conocer preocupaciones, problemas, ideas y sentimientos, interactuando constantemente con las personas.

En el escenario asistencial (21) hay que tener presente el contexto puesto que facilita el tránsito del proceso salud-en- 
fermedad, involucrando a la familia como un acto de humanización de la atención en salud (41). Por consiguiente, no solo se debe pensar en un cuidado humano sino está presente la familia; por ello esta necesidad particular indica que este análisis deba efectuarse desde una perspectiva de satisfacción y felicidad de la condición humana.

Hay que mencionar además, que la humanización de la atención en salud significa cuidar de las personas colectivamente, con responsabilidad, compromiso y ética, ayudándoles a vencer sus limitaciones, promoviendo su automanejo (42) repensando, replanteando y reinventando las formas cómo las cuidamos, las tratamos y las respetamos en los servicios de salud.

\section{Paso 7. Identificar un caso modelo del concepto.}

Conviene indicar que se parte de la iniciativa "Unamos Humanos" del Grupo de Cuidado de Facultad de Enfermería de la Universidad Nacional de Colombia 2016-2018.

En el trabajo de acompañamiento que realiza el grupo de cuidado, se encontró pertinente apoyar la iniciativa "unamos humanos" como una invitación a vincular estudiantes, egresados y profesores que participan en las reuniones de grupo de cuidado con un esquema de trabajo colaborativo y de interacción humana. "Unamos humanos" se constituyó en 2016 como un grupo estudiantil que se registró en la Dirección de Bienestar bajo el proyecto Bienestar espiritual con el uso de herramientas sanadoras. Este caso modelo de trabajo del grupo permitió evidenciar en la práctica, la utilización de herramientas vivenciales como la meditación, la biodanza y el Reiki, las cuales facilitan procesos de auto reflexión y apropiación social del conocimiento en entornos relevantes, como lo es en el universitario.

Se debe destacar que este proyecto fue ganador de apoyo por parte de la facultad; igualmente se contó con el grupo de estudiantes y egresados que ofrecieron sus saberes y vivencias aportando de manera concreta sus conocimientos respecto a las formas que favorecen la humanización en salud. Los profesionales de la salud y los estudiantes de enfermería se constituyeron así en agentes promotores de prácticas que humanizan la atención, ya que, al reconocer el aporte de estas herramientas sanadoras, se logra como beneficio fomentar los valores y principios de una práctica comprometida con los seres humanos, con hacer el bien, con colaborar y ser solidario. En las evaluaciones formativas, al finalizar este proyecto, se logró documentar las percepciones de los participantes en su formación integral en la Facultad de Enfermería. Acciones que permitieron vivir momentos de interacción que no se olvidan, cultivando su bienestar espiritual; al mismo tiempo se preparan de la mejor manera para cuidar a otros desde una perspectiva de humanización de la atención en salud. Se espera que este modelo pueda ser multiplicado en otros escenarios de formación de profesionales de la salud y de la práctica del cuidado.

Finalmente, el modelo de caso anteriormente expuesto puede enmarcarse en la concepción teórica de Watson para quien la enfermería es una ciencia, así como un arte del cuidado transpersonal desde una perspectiva fenomenológica que incluye aspectos del vínculo transaccional entre personas, con el fin de dignificar al ser humano y crear momentos de humanización de la atención (43).

\section{Discusión}

En este estudio se propuso aclarar el concepto de humanización de la atención en salud aplicando el método de análisis propuesto por Rodgers (13) siguiendo cada uno de los pasos, enriquecido por análisis de estudios ubicados en diferentes bases de datos.

Es importante señalar que en los estudios examinados se pudo evidenciar cómo el concepto de humanización de la atención en salud se discute desde diferentes ámbitos académicos, clínicos y comunitarios, destacándose la presencia de un patrón ético, propio de la atención en salud, en donde factores como el trabajo en equipo y los procesos de comunicación continua y efectiva son indispensables que se manejen adecuadamente por parte de los diferentes actores de la atención en salud.

Como se ha planteado el concepto se desarrolla en diferentes escenarios de la atención en salud; sin embargo, se presentan barreras relacionadas con la humanización en el servicio de urgencias, tales como la ausencia de capacitación en competencias sobre la compasión, la desinformación en el área de especialización y el aumento de la razón enfermero paciente (44). En el escenario de la Unidad de Cuidado Intensivo la humanización involucra componentes técnicos, tecnológicos y organizativos, involucrando esencialmente sus dimensiones políticas y filosóficas que le confieren un carácter ético, solidario y humanizado en acciones de cuidado (45).

Otro rasgo a tener en cuenta, es que desde el escenario académico, se identifican elementos teórico-prácticos que se han construido sobre la enseñanza de la humanización en el contexto de salud, tornándose imprescindibles, fomentando mayores inversiones en la construcción efectiva de nuevos modos de cuidar (46), lo que responde a un camino epistemológico del cuidado desde sus orígenes hasta la actualidad.

El atributo predominante presentado fue el de la comunicación tanto en el ámbito profesional como en el organizacional con el fin de propender por la humanización en la atención en salud (47). En la mayoría de los estudios el enfoque predominante fue la ética entendiéndose en función de factores relacionados con: la compasión, la responsabilidad y el hacer al otro lo mejor, visibilizando la ontología en el acto de cuidado y atención de los humanos como un eje fundamental.

Estudiar la humanización de los servicios de salud como concepto, permitió entender las implicaciones que tiene en la enfermería y las ciencias de la salud, a partir de la formación, la práctica, así como de la investigación desde 


\section{Scientific Journal of Nursing}

el actuar profesional y disciplinar, tal como lo menciona Azeredo quien convoca a visibilizar la atención humanizada desde la política pública, basada en el respeto y la lucha por los derechos de los pacientes, previniendo la violencia en la atención en salud de los humanos (48).

Como consecuencia del análisis del concepto se puede deducir que las habilidades blandas son un eje norteador para el cuidado humanizado; concepto que coincide con el estudio de Ortiz et al., quienes describen que entre los factores relacionados con la falta de humanización están: la ausencia de capacitación en competencia de compasión, mindfullness y habilidades blandas; agregan la desinformación en el área de especialización y el aumento de la razón enfermero-paciente (44). Como se puede percibir este panorama permite inferir la necesidad de se tengan en cuenta para futuros estudios investigativos, aplicando metodologías innovadoras y contextualizadas a las realidades de los profesionales de la salud en diferentes servicios de atención.

\section{Conclusiones}

- El análisis del concepto permitió presentar una aproximación teórica y reflexiva de algunos elementos éticos, epistemológicos y metodológi$\cos$ de la humanización de la atención en salud, como un concepto relevante en ciencias de la salud.

- Desde la perspectiva de la Enfermería como disciplina y ciencia enfocada al cuidado y la humanización de la atención en salud se constituye en un foco para direccionar sus acciones en beneficio del bienestar y la buena práctica, que muestren un desarrollo del concepto y un camino epistemológico inmerso en la práctica del cuidado. Aunque el concepto de humanización de la atención se puede analizar desde diferentes perspecti- vas, la metodología del análisis de este concepto es una perspectiva útil para considerar a la humanización de la atención en salud como un eje orientador de políticas de mejoramiento y calidad de la atención, así como en la promoción de prácticas que faciliten el trabajo colaborativo, las habilidades blandas, la comunicación, una ética del cuidado desde lo ontológico y el desarrollo humano, siempre respetando los derechos humanos.

- Con lo anteriormente expuesto se plantea la oportunidad de contribuir al cierre de la brecha entre la teoría y la práctica, al mostrar los resultados de proyectos de trabajo colaborativo entre estudiantes y profesores, que pueden promover una práctica de enfermería con enfoque transformador para la humanización en servicios básicos (49). Además, se debe precisar que es de alta complejidad, al entrar en juego el aprendizaje, apropiándose de momentos de interacción significativa.

- También, se evidencian metodologías de investigación, en donde se revisan la literatura, estudios exploratorios y descriptivos relacionados con el concepto de humanización de la atención en salud; no obstante, emerge la necesidad desde el método de promover en el nivel de conocimiento estudios futuros mixtos, de correlación, control y predicción para contar con niveles de evidencia científica, que permitan la traslación del conocimiento a la práctica, y así propiciar una atención en los servicios de salud innovadores, contextualizados, de vanguardia, seguros y con un modelo de atención centrado en la persona y su familia.

\section{Conflicto de intereses}

Los auotres delcaran no tenen ningun conflicto de intereses

\section{Referencias Bibliográficas}

1. Correa-Zambrano ML. La humanización de la atención en los servicios de salud: un asunto de cuidado. Rev Cuid. 2016;7(1):1227-31.

2. Stein-Backes D, Santos-Koerich M, Lorenzini-Erdmann A. Humanizando o cuidado pela valorização do ser humano : re-significação de valores e princípios pelos profissionais da saúde. Rev Latino-Americana Enferm I. 2007;15(1).

3. Linero MI, Mendivelso D, Raba AM, Duarte D. Anexo técnico: Calidad y humanización de la atención en salud a mujeres gestantes, niñas, niños y adolescentes Dirección de Promoción y Prevención Grupo Curso de Vida. 2014.

4. Walker LO, Avant KC. Strategies for theory construction in nursing. Upper Saddle River, N.J. : Pearson/Prentice Hall; 2005.

5. Jacox A. Theory Construction in Nursing: An Overview. Nurs Res. 1974;23(1):4-13.

6. $\quad$ Price B. Chronic illness experience Reading.pdf. J Adv Nurs. 1996;24:275-9.

7. Morse J. Exploring the theoretical basis of nursing using advanced techniques of concept analysis. Adv nursng sience. 1995;17(3):31-46.

8. Rodgers BL, Knafl KA. Concept Development in Nursing: Foundations, Techniques, and Applications. 2nd ed. Saunders, editor. 2000. 85 p. 
9. Canizales-Caicedo MT. Marco Tulio Canizales C tesis Manizalez. Universidad Catolica de Manizalez; 2016.

10. Oviedo AD, Delgado IAV, Licona JFM. Habilidades sociales de comunicación en el cuidado humanizado de enfermería: Un diagnóstico para una intervención socioeducativa. Esc Anna Nery. 2020;24(2):1-7.

11. Correa-Zambrano ML.Humanización de la atención en los servicios de salud: un asunto de cuidado. Rev Cuid. 2016;1(7):1227-31.

12. Ugarte Chang JA. Cuidado humanizado de enfermería según opinión de las personas viviendo con Virus de la Inmunodeficiencia Humana en estado de SIDA. Rev Cienc y Arte Enfermería. 2017;2(1):40-6.

13. Rodgers Beth. Knafl Kathleena. Concept Development in Nursing. Second Edi. Saunders.., editor. 2000.85 p.

14. Campiño-Valederrama SM, Duque PA, Cardozo-Arias VH. Percepción del paciente hospitalizado sobre el cuidado brindado por estudiantes de enfermería. Univ y Salud. 2019;21(3):215-25.

15. Pimenta De Oliveira C, Henriqueta M, Kruse L. A humanização e seus múltiplos discursos-análise a partir da REBEn. Vol. 59, Rev Bras Enferm. 2006.

16. Romero-Massa E, Contreras-Méndez IM, Moncada-Serrano A. Relación Entre Cuidado Humanizado Por Enfermeria Con La Hospitalización De Pacientes. Hacia la promoción la salud. 2016;21(1):26-36.

17. Sousa KHJF, Damasceno CKCS, Almeida CAPL, Magalhães JM, Ferreira M de A. Humanização nos serviços de urgência e emergência: contribuições para o cuidado de enfermagem. Rev Gauch Enferm. 2019 Jun 10;40:e20180263.

18. Troncoso MP, Suazo SV. Cuidado humanizado: Um desafio para enfermeiras nos serviços hospitalares. ACTA Paul Enferm. 2007;20(4):499-503.

19. Allande-Cussó R, Navarro-Navarro C, Porcel-Gálvez AM. Humanized care in a death for COVID-19: A case study. Enferm Clin [Internet]. 2021;31:S62-7. Available from: https://doi.org/10.1016/j.enfcli.2020.05.018

20. Posada M, Mora B. Interpretación de la teoría de Kristen Swanson para un cuidado humanizado. Metas Enferm. 2014;17(3):69-75.

21. Gonçalves De Oliveira BR, Collet N, Viera CS. A Humanização na assistência à saúde. Rev Latino-am Enferm [Internet]. 2006;14(2):277-84. Available from: https://www.scielo.br/j/rlae/a/dvLXxtBqr9dNQzjN8HWR3cg/?lang=pt\&format $=$ pdf

22. Arriaga-García A, Obregón-De La Torre M. Conocimientos y actitudes sobre el cuidado humanizado en enfermeras de Lima. CASUS Rev Investig y Casos en Salud. 2019;4(2):102-10.

23. Chernicharo I de M, Freitas FD da S de, Ferreira M de A. Humanização no cuidado de enfermagem: contribuição ao debate sobre a Política Nacional de Humanização. Rev Bras Enferm. 2013;66(4):564-70.

24. Monje P, Miranda P, Oyarzún J, Seguel F, Flores E. Perception of humanized nursing care by hospitalized users. Cienc y Enferm. 2018;24(5):1-10. Disponible en: http://dx.doi.org/10.4067/s0717-95532018000100205

25. Pacheco de Araújo F, Ferreira M de A. Representações sociais sobre humanização do cuidado: implicações éticas e morais. Rev Bras Enferm. 2011;64(2):287-93.

26. Marques ES, de Moraes CL, Hasselmann MH, Deslandes SF, Reichenheim ME. Violence against women, children, and adolescents during the COVID-19 pandemic: Overview, contributing factors, and mitigating measures. Cad Saude Publica. 2020;36(4).

27. Nora CRD, Junges JR. Humanization policy in primary health care: A systematic review [Internet]. Vol. 47, Revista de Saude Publica. 2013. p. 1186-200. Available from: https://dx.doi.org/10.1590\%2FS0034-8910.2013047004581

28. Mongiovi VG, dos Anjos Rde C, Soares SB, Lago-Falcão TM. Conceptual reflections on health humanization: conception of nurses from Intensive Care Units. Rev Bras Enferm. 2014;67(2):306-11. Disponible en: https://doi. org/10.5935/0034-7167.20140042

29. Akiko-Komura L. A dimensão subjectiva do profissional na humanização da assistência à saúde: uma reflexão. Rev Esc Enferm USP. 2004;38(1):13-20.

30. McDermit M. Análisis conceptual de la enfermería. Bol la Of Sanit Panam. 1974;76(1):64-71.

31. Collet N, Rozendo CA. Humanização e trabalho na enfermagem. Rev Bras Enferm. 2003;56(2):189-92.

32. Casate JC, Correa A. Humanização do atendimento em saúde: conhecimento veiculado na literatura brasileira de enfermagem1 humanização do atendimento em saúde: conhecimento veiculado na literatura brasileira de enfermagem. Rev Latino-am Enferm. 2005;13(1):105-11. 


\section{Scientific Journal of Nursing}

33. Da Nóbrega Morais GS, Da Costa SFG, Fontes WD, Carneiro AD. Communication as a basic instrument in providing humanized nursing care for the hospitalized patient. ACTA Paul Enferm. 2009;22(3):323-7.

34. Ribeiro-Ferreira L, Artmann E. Pronouncements on humanization: Professionals and users in a complex health institution. Cienc e Saude Coletiva. 2018;23(5):1437-50. Disponible en: https://doi.org/10.1590/1413-81232018235.14162016 35. Cruz C. La naturaleza del cuidado humanizado. Enfermería Cuid Humaniz. 2020;9(1):21-32. Disponible en: https:// doi.org/10.22235/ech.v9i1.2146

36. Morales-Asensio JM. Barreras para la implementación de modelos de práctica avanzada en España. Metas de Enfermería [Internet]. 2012;15(6):26-32. Available from: https://medes.com/publication/75689

37. Freitas FD, Ferreira MA. Saberes de estudantes de enfermagem sobre a humanização. Rev Bras Enferm. 2016;69(2):282-289.

38. Zuñiga M. Humanizar la Salud una propuesta desde el caribe colombiano. In: Capitulo 3 [Internet]. Universida. Barranquilla; 2016. p. 25-30. Available from: https://books.google.com.co/books?hl=es\&lr=\&id=LtJCDwAAQBA-

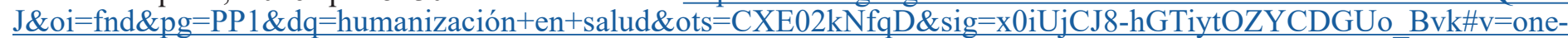
page\&q\&f=false

39. Vara MA, Fabrellas N. Análisis de concepto: gestión enfermera de la demandaConcept analysis: Nurse demand management. Atención Primaria. 2019;51(4):230-5.

40. Pallares S. Enfermería: Integración del cuidado y el amor. Una perspectiva humana. Salud Uninorte. 2009;25(1):172178.

41. Pimenta C, Luce MH. A humanização e seus múltiplos discursos-análise a partir da REBEn. Rev Bras Enferm. 2006;59(1):78-83.

42. Garnett A, Ploeg J, Markle-Reid M, Strachan PH. Self-Management of Multiple Chronic Conditions by Community-Dwelling Older Adults: A Concept Analysis. SAGE Open Nurs. 2018;4:1-16.

43. Fawcett J, DeSanto-Madeya S. Contemporary Nursing Knowledge: Analysis and Evaluation of Nursing Models and Theories. 3, editor. 2013.

44. Pabón-Ortíz EM, Mora-Cruz JV-D, Castiblanco-Montañez RA, Buitrago-Buitrago CY. Estrategias para fortalecer la humanización de los servicios en salud en urgencias. Rev. cienc. cuidad. 2021; 18(1):94-104.

45. Calvetti A, Crecencia H, Zamberlan C, Cecagno D, dos Santos S, Bergmann MR. Integralidade e humanização na gestão do cuidado de enfermagem na Unidade de Terapia Intensiva. Rev da Esc Enferm da USP. 2016;50(5):817-23.

46. Casate JC, Corr AK. A humanização do cuidado na formação dos profissionais de saúde nos cursos de graduação. Rev Esc Enferm USP. 2012;46(1):219-26.

47. Saucedo IG. La comunicación enfermera-paciente: una estrategia para la humanización del cuidado Nurse-patient communication: A strategy in humanizing care. Rev Enferm Inst Mex Seguro Soc. 2016;24(2):85-6.

48. AzeredoYN, Schraiber LB. Autoridad, poder y violencia: un estudio sobre humanización en salud. Interface-Comunicação, Saúde, Educação. 2021; 25: e19083849. https://doi.org/10.1590/Interface.190838

49. Soares-Rezende-Lopes MT, Gomes-Labegalini CM, Antoniassi-Baldissera VD. Educar para humanizar: o papel transformador da educação permanente na humanização da atenção básica. Rev enferm UERJ. 2017; 25:e26278. https://doi. org/10.12957/reuerj.2017.26278 